\title{
Il Museo Internazionale delle Ceramiche in Faenza: il sistema delle misurazioni
}

\author{
Anna Maria Nati*
}

Ricevuto il 3 Settembre 2012

Accettato il 13 Luglio 2012

\begin{abstract}
The Museo Internazionale delle Ceramiche in Faenza: the measurement system The author - through information derived from several written documents, website sources and interviews as well as from proposals from the italian and foreign doctrine - examines the most significant measurements of the Museo Internazionale delle Ceramiche in Faenza.

The aims of this survey are three:

- $\quad$ to verify to what extent the budget meet the standards indicated by academic scholars in Business Administration;

- $\quad$ to ascertain how problems originated by the cost accounting practice have been resolved and which are the connected aims;

- $\quad$ to illustrate the performance indicators utilized, including their specific aims.
\end{abstract}

Keywords: Budget, Costs, Indicators.

\section{Presentazione del Museo}

Il Museo Internazionale delle Ceramiche in Faenza (M.I.C) ha la sede espositiva nel bel viale che dalla Stazione Ferroviaria permette di raggiungere la più importante piazza, di gran pregio architettonico, della operosa città romagnola. La sua tradizione plurisecolare nella lavorazione della ceramica, che ancor oggi ha un ruolo di primo piano nell'economia cittadina, è testimoniata anche dal fatto che il suo toponimo è sinonimo di maiolica in più lingue; basti pensare che il termine francese faïence significa faenza, faentina, maiolica.

Fondato nel 1908 - in occasione del terzo centenario della nascita di E.

*Professore associato in Economia Aziendale presso il Dipartimento di Scienze Aziendali - Università degli Studi di Firenze. Anna Maria Nati curerà la corrispondenza con il Comitato di indirizzo (annamaria.nati@unifi.it). 
Torricelli, scienziato faentino inventore del barometro (AA.VV., 2008, p. 45) - il M.I.C, sorto inizialmente come Ente morale e poi divenuto Museo civico, nel 2001 è stato affidato in gestione a una Fondazione di diritto privato, appositamente costituita da enti pubblici e privati, che ha sede legale in Faenza in Via Campidori, n. 2. Alla Fondazione, che si prefigge di valorizzare la più importante istituzione culturale della città e di promuovere lo sviluppo economico del territorio potenziando le interrelazioni con i settori economici di riferimento (primo fra tutti quello del turismo), partecipano il Comune di Faenza, la Provincia e la Camera di Commercio di Ravenna, istituzioni private e aziende per un totale di 19 soci (Del Sordo et al., 2010, p. 69 e ss.).

Per ragioni tecniche, economiche e di opportunità sociale, la Fondazione gestisce in regime di concessione il Museo; il concedente Comune di Faenza, al cui Sindaco spetta la nomina del Presidente della Fondazione, conserva la proprietà del patrimonio immobiliare e delle collezioni museali presenti nel giorno (15.06.2001) della delibera del Consiglio Comunale relativa alla costituzione della Fondazione M.I.C.

Il Museo fa parte degli Istituti Culturali di Faenza, aderisce - tramite apposita convenzione - al Sistema Museale della Provincia di Ravenna ed è riconosciuto dalla Regione Emilia Romagna "Istituto per i beni artistici culturali e naturali".

La mission (Bernardi 2004; Rocchi 2004; Rocchi 2007) del M.I.C è compiutamente indicata nella Carta dei servizi che così recita: «Il Museo è un'istituzione permanente senza fini di lucro, al servizio della comunità, aperta al pubblico, il cui scopo primario è quello di custodire, conservare, valorizzare e promuovere lo studio e la conoscenza delle proprie collezioni ed in generale del patrimonio culturale e storico dell'arte ceramica, in ambito nazionale e internazionale, al fine sia di preservare la memoria e sollecitare la curiosità nei confronti del passato sia di promuovere la conoscenza di strumenti critici utili alla migliore comprensione del presente».

Coerentemente con la mission, a partire dalla sua formulazione originaria fino ad arrivare a quella odierna sopra riportata, sono stati via via definiti degli obiettivi che impegnano la direzione nella ricerca di un maggior sviluppo delle azioni volte sia alla conservazione, tutela e valorizzazione del patrimonio museale sia alla promozione e diffusione della cultura.

Parallelamente all'evoluzione degli obiettivi, si osservano cambiamenti nelle attività poste in essere per il loro perseguimento. Ne costituiscono esempi significativi le «[...] iniziative di istruzione e formazione, di ricerca 
e restauro in ambito ceramico, di divulgazione attraverso opportune attività editoriali» 1 .

Gli scopi e le azioni intraprese sono stati oggetto di comunicazione per diffondere la conoscenza sullo svolgimento della gestione; più precisamente, per poter decidere in modo razionale, per misurare la performance aziendale e per valutare il grado di soddisfacimento delle aspettative di alcuni stakeholder.

Tutto ciò dimostra lo stretto legame tra mission e accountability (Matacena, 2007, p. 19 e segg.) $)^{2}$ nel senso che le finalità istituzionali, che ispirano gli organi direttivi, si traducono in comportamenti conformi ad esse e le une e gli altri si "trasformano" in informazioni, sia pur in modo non del tutto organico e completo.

In sintonia con la mission, il M.I.C pone in essere una vasta gamma di attività tra le quali ci preme segnalare le seguenti:

- attività espositive: mostra permanente che fa parte della più grande raccolta al mondo di opere in ceramica, provenienti soprattutto da donazioni, e che testimonia, a partire dal V millennio A.C. fino ai giorni nostri, la cultura di questa espressione artistica nei cinque continenti; mostre temporanee sia all'interno (e talora dedicate ad altre arti figurative) che all'esterno del Museo (il cosiddetto M.I.C fuori dal M.I.C che annovera esposizioni talvolta itineranti come quella, detta Musica di smalto, accolta in più continenti); prestiti ad esposizioni nazionali e internazionali; la mostra collegata al concorso internazionale della ceramica d'arte contemporanea;

- restauro, affidato a un laboratorio che si occupa dell'intera attività conservativa delle opere che, in gran parte, richiedono interventi preventivi come la collocazione in ambienti adatti ad accoglierle. A ciò si aggiunge l'esecuzione di restauri, a titolo oneroso o gratuito, richiesti da committenti esterni, privati e pubblici (è evidente che, quanto meno nel primo caso, è importante rilevare il costo dell'intervento per fissarne il prezzo);

- ricerca scientifica svolta anche in collaborazione con ceramologi e istituti culturali operanti in Italia e all'estero;

- attività didattiche e formative quali: i corsi destinati agli specialisti del restauro; il laboratorio Giocare con l'Arte dove i protagonisti dell'esecuzione

\footnotetext{
${ }^{1}$ Queste attività, inserite nello Statuto a seguito della sua modifica avvenuta nel 2007, denunciano chiaramente l'esigenza di un sistema di misurazioni flessibile, cioè capace di estendere il suo raggio di azione a nuovi interventi che in futuro si decidesse di realizzare.

${ }^{2}$ Sui vari aspetti dell'accountability nei musei, tra gli altri, si veda: Carnegie e Wolnizer, 1996; Catalfo e Molina, 2004; Matacena, 2007, p. 25 e ss.; Monteduro, 2004; Rentschler e Potter, 1996; Sibilio, 2007, p. 54 e ss.; Sibilio Parri, 2011.
} 
delle varie tecniche ceramiche sono i bambini; corsi di formazione e aggiornamento rivolti agli adulti e ai docenti delle scuole di ogni ordine e grado; percorsi storici e tematici attraverso un servizio di visite guidate, in sinergia con i programmi svolti in classe dagli insegnanti e destinati a un pubblico scolastico di varia provenienza territoriale; le Domeniche x bambini + adulti; attività di tutoraggio di stagisti e tirocinanti;

- gestione di una fototeca della maiolica italiana, strumento di documentazione iconografica delle opere presenti nel M.I.C e nelle collezioni, private e pubbliche, di tutto il mondo, nonché di una biblioteca specializzata, soprattutto sull'arte della ceramica, che, con circa 62.000 opere, è nel settore la più grande del nostro pianeta;

- occasioni di intrattenimento, in primis quella musicale, talora precedute da percorsi a tema fra le raccolte del Museo;

- attività editoriale e di comunicazione tra cui spicca, rispettivamente, la pubblicazione della rivista "Faenza", nata nel 1913, e la gestione del sito web che ha ricevuto il plauso del Ministero per i Beni e le Attività Culturali;

- incontri e collaborazioni con "addetti ai lavori” di nazionalità straniera e partecipazione a progetti nazionali ed europei, talora in veste di lead partner (AA.VV., 2008, p. 5 e ss.);

- manifestazioni anche al di fuori delle attività museali come, ad esempio, congressi nel campo della medicina.

Da sottolineare infine, ed è un risultato coerente con la mission del $\mathrm{Mu}-$ seo, il riconoscimento del M.I.C quale Monumento Messaggero di una Cultura di Pace presso il Segretariato generale dell'Unesco a Parigi.

Anche solo considerando le attività sopra descritte, emerge evidente la necessità di un sistema di misurazioni ampio e capace di tradurre in corretti valori di sintesi, monetari e non, lo svolgimento di attività che spesso sono molto diverse tra di loro.

Di rilievo è anche il ricorso all'outsourcing nella veste di contratti sia a progetto (come quello per la realizzazione del catalogo elettronico della biblioteca) che di tipo "professionale” relativi, ad esempio, ai servizi di accoglienza e di sorveglianza delle sale. Ovviamente, al pari delle attività svolte internamente, anche quelle esternalizzate devono essere oggetto di misurazione e rispettare i due fondamentali criteri dell'efficienza e dell'efficacia.

Di particolare interesse sono i rapporti con enti locali territoriali per quanto concerne il personale e le fonti di finanziamento ${ }^{3}$.

\footnotetext{
${ }^{3}$ Sul finanziamento dei musei, tra gli altri, si consulti: Ferretti, 2007, p. 233 e ss.; Zangrandi, 2007, p. 93 e ss.
} 
Più precisamente, nel M.I.C presta la propria opera il personale "distaccato” dal Comune di Faenza e dalla Regione Emilia Romagna (trattasi, rispettivamente, di 13 e 2 dipendenti ${ }^{4}$ ).

Quanto ai finanziamenti, emergono per la loro entità i contributi versati dal Comune di Faenza a sostegno sia dell'attività istituzionale del Museo sia di particolari spese. Sempre diretti a supportare attività specifiche o progetti ben precisi sono i finanziamenti provenienti dalla Regione Emilia Romagna che si aggiungono a quelli ottenuti dalla Provincia di Ravenna che finanzia anche la suddetta attività. Inoltre, sono da considerarsi una forma indiretta di finanziamento sia la remunerazione, a carico dei rispettivi enti locali, del personale comunale e regionale "distaccato" presso il M.I.C sia le attrezzature per la biblioteca ottenute in comodato da detta Provincia.

Vi sono, poi, altri finanziatori: sponsor tecnici, affiancatori esterni istituzionali o imprenditoriali (come il Consorzio Romagna Acque), banche e fondazioni bancarie, soci della Fondazione quando erogano contributi e privati cittadini.

Infine, nell'ampio e diversificato panorama delle fonti di finanziamento, non mancano le più tradizionali forme di provvista dei capitali quale, ad esempio, l'autofinanziamento essendo inibita, per Statuto, la distribuzione di utili da impiegare invece, come precisa l'art. 3 comma 4), nella «[...] realizzazione delle attività istituzionali e di quelle ad esse direttamente connesse».

Sono dunque numerosi e di varia natura i meccanismi di finanziamento che, in quanto tali, sono riconducibili a portatori di specifiche esigenze conoscitive a cui il Museo risponde con un'ampia e variegata informativa.

Il controllo di gestione, con le sue tipiche rilevazioni, è un processo a cui il M.I.C riserva un' esplicita attenzione. Nel Regolamento del Museo, infatti, si parla di individuazione delle entrate specifiche che si prevede di destinare ad attività dello stesso, di programmazione, di successivo monitoraggio dei programmi, della costante riformulazione, durante l'anno e a consuntivo, degli obiettivi e dell'impiego di appropriati indicatori della qualità.

Il richiamo alla qualità è presente anche nella Carta dei servizi - documento, come il precedente, molto dettagliato - dove, oltre a prospettarne il miglioramento tramite la collaborazione degli utenti, viene considerata non disgiunta dai criteri di efficacia e di efficienza, posti alla base dell'erogazione dei servizi museali, che sappiamo richiedono precisi obiet-

\footnotetext{
${ }^{4}$ A questi si aggiunge un folto numero di collaboratori esterni che, a vario titolo, operano nel M.I.C a fronte di soli 3 dipendenti della Fondazione.
} 
tivi e adeguate verifiche e, dunque, ancora una volta, apposite misurazioni ${ }^{5}$. Similmente dicasi per la produttività sulla quale produce un effetto positivo l'atteggiamento del vertice aziendale volto a far sì che ogni operatore senta suo il Museo.

Scendendo nel particolare, un altro esplicito richiamo all'efficienza e all'efficacia è rintracciabile nella relazione al Bilancio preventivo del 2011 dove si afferma che nella predisposizione di detto documento «[...] si è continuato a perseguire l'obiettivo di una attenta razionalizzazione della spesa e di un concreto controllo di gestione teso ad evitare costi superflui rispettando nel contempo il piano di investimenti previsti e gli interventi nel settore sicurezza programmati dalla Fondazione».

La struttura organizzativa del M.I.C (tab.1) presenta una certa snellezza se si considera che in posizione subordinata al Consiglio di Amministrazione si trovano organo collocati su due livelli gerarchici e che taluni ruoli sono ricoperti da una sola persona. Ciò facilita e rende più veloce il flusso informativo con impliciti riflessi positivi sul controllo di gestione.

Da segnalare, poi, le modifiche apportate a detta struttura nel 2011. Trattasi, più precisamente, del passaggio dell'organo addetto all'informatica alle dipendenze del Direttore, e non più del Segretario generale, vista la sua prevalente mansione di comunicazione; della scomparsa dall'organigramma del Conservatore del ' 900 dal momento che il nuovo Direttore è specializzato in arte contemporanea; dell' assegnazione al Segretario generale di tutti i compiti delegabili dal Consiglio di Amministrazione in materia di sicurezza ai sensi del D.Lgs. 81/2008.

Per finire, merita ricordare che il Museo si è, formalmente ed esplicitamente, impegnato a tutelare la chiarezza e la completezza delle informazioni destinate ai vari interlocutori nonché la trasparenza e la veridicità delle scritture contabili e del bilancio.

\footnotetext{
${ }^{5}$ Sempre nella Carta dei servizi si precisa che il «[...] Museo adegua il proprio ordinamento e funzionamento agli standard e obiettivi di qualità previsti dal Decreto Ministeriale del 10 maggio 2001 e dalla deliberazione della Giunta Regionale n. 309/2003». Vengono poi indicati per ogni "dimensione" (ad esempio, programmazione e controllo) uno o più indicatori di tipo descrittivo con i relativi standard di riferimento garantiti (continuando con l'esempio, approvazione dei documenti programmatici nei termini previsti). Resta da dire che il M.I.C ha ottenuto il riconoscimento, da parte della Regione Emilia Romagna, di Museo di qualità divenendo, così, un istituto culturale accreditato.
} 
Tab. 1 - Organigramma del M.I.C

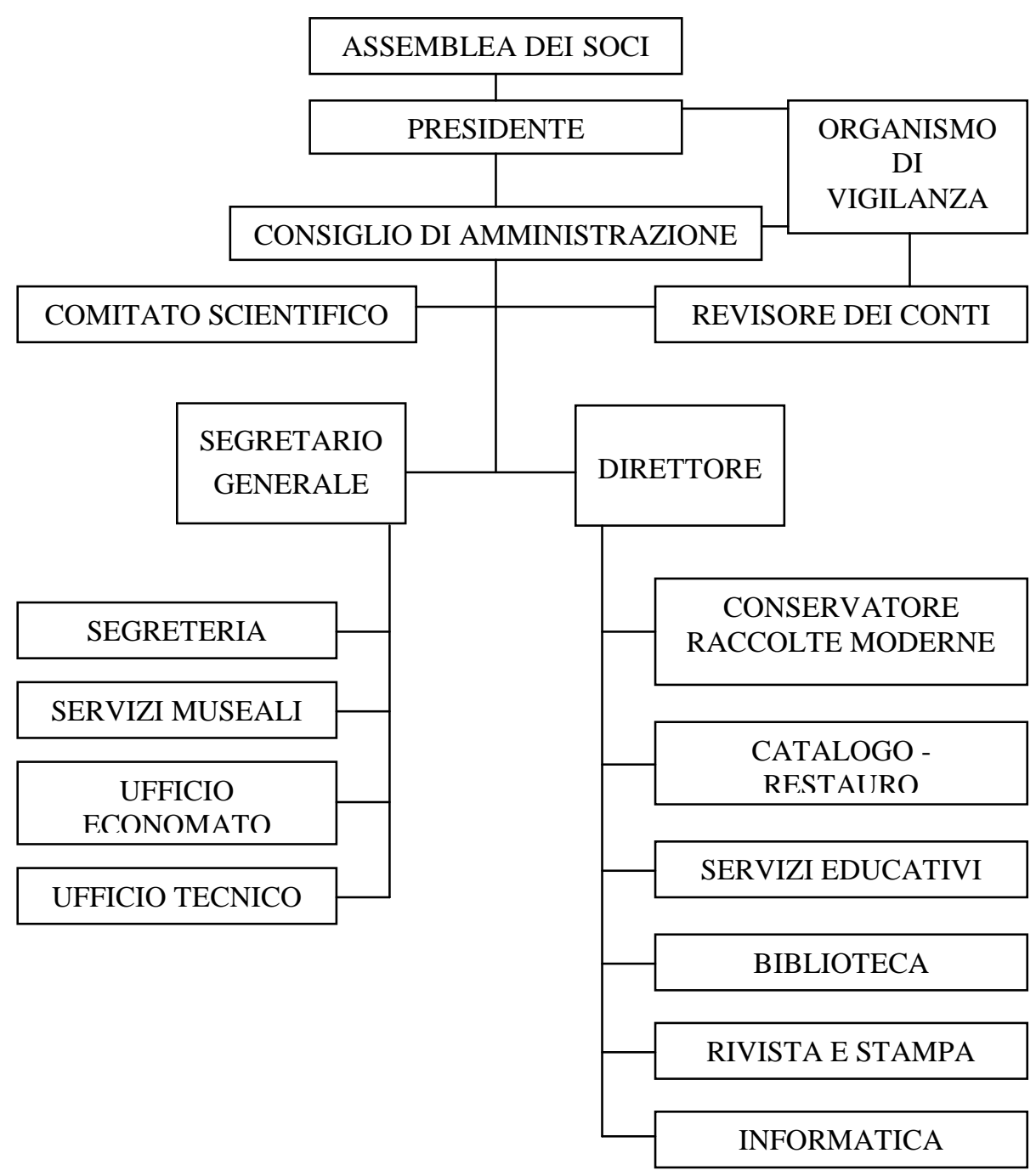

\section{Le domande di ricerca}

I musei necessitano di un sistema di misurazioni della loro performance nei vari aspetti in cui essa si esplicita (efficienza gestionale, efficacia gestionale e sociale, produttività, qualità e via dicendo) e nei momenti in cui va programmata, monitorata, controllata e rendicontata. 
È pertanto chiaro che detto sistema deve produrre informazioni:

- per la pianificazione strategica, il controllo di gestione, la periodica rilevazione dei fatti amministrativi, la redazione del bilancio di esercizio e del bilancio sociale;

- di diversa natura, riferite cioè alla dimensione a cui sono collegate (istituzionale, culturale, economica, finanziaria e patrimoniale) e alla veste con cui si presentano (quantitativo-monetaria, quantitativa e qualitativa);

- destinate sia a soggetti interni sia a interlocutori esterni (fruitori, comunità culturale, finanziatori, etc.).

Risulta così confermata la multidimensionalità di ogni informazione ${ }^{6}$.

Ciò premesso, ricordiamo che il Museo Internazionale delle Ceramiche in Faenza riserva grande attenzione all'efficacia, all'efficienza e alla qualità delle sue prestazioni. Pertanto, è legittimo chiedersi in che modo queste finalità si riflettono nelle misurazioni che il Museo pone in essere.

In altre parole, le domande di ricerca alle quali tenteremo di rispondere possono così formularsi:

1. il budget è un programma globale di gestione espresso in termini descrittivi ma, soprattutto, economici, finanziari e patrimoniali, flessibile, articolato in senso spaziale e temporale, che guida e responsabilizza gli operatori aziendali verso obiettivi di breve periodo coerenti e strumentali a quelli definiti nell'ambito del piano strategico (Brusa, 2000, p. 84 e ss.; Horngren et al., 2002, p. 468 e ss.; McLaney e Atrill, 2002, p. 355 e ss.); detto questo, il budget assume tali connotazioni anche nel M.I.C?;

2. nell'impostare un sistema di rilevazione dei costi occorre, alla luce delle esigenze conoscitive da soddisfare, definire i caratteri della/e "figu$\mathrm{ra} / \mathrm{e}$ " di costo che si intende costruire e risolvere alcuni problemi metodologici (Brusa, 1995, p. 1 e ss.; Cinquini, 2008, p. 75 e ss.; Garrison e Noreen, 2004, p. 29 e ss.; Ruggieri, 2004, p. 33 e ss.): da qui, la domanda: quali scelte ha fatto il M.I.C e per quali scopi?;

3. le informazioni economico-finanziarie, in qualunque azienda e ancor più nei musei, sono insufficienti ad esprimere e valutare il complessivo andamento della gestione. È quindi indispensabile integrarle con indicatori quantitativo-non monetari e qualitativi (Agliati, 2003, p. 129 e ss.; Brunetti, 1993; Giacinti, 2010; Ittner e Larcker, 2003; Zappa, 1957, pp. 883-884); ciò puntualizzato, quali indicatori non financial vengono utilizzati dal M.I.C e per quali finalità?

Budget, costi e indicatori non monetari sono inquadrabili in un'ottica si-

\footnotetext{
${ }^{6}$ La multidimensionalità caratterizza anche il sistema informativo che deve, altresì, essere “integrale” e “integrato” (Sibilio, 2004, pp. 61-62).
} 
stemica dati i nessi che li legano tra loro. Più precisamente, il budget economico, che è quello che analizzeremo, accoglie costi e ricavi preventivi i quali, poi, vanno confrontati con quelli consuntivi onde appurare i motivi di eventuali scostamenti. A loro volta, taluni indicatori fisico-tecnici e qualitativi evidenziano le cause del sorgere e/o dell'ammontare di dati costi e ricavi.

Ecco, allora, che il rapporto causa-effetto e il riferimento sia al controllo di gestione che alla misurazione della performance diventano elementi unificanti del sottosistema di misurazioni oggetto della nostra analisi.

È infine doveroso puntualizzare che, nel dare risposta ai quesiti sopra indicati, ci siamo avvalsi, come in precedenza, di quanto appreso dai documenti forniti, dalle interviste a più operatori del M.I.C e dal già menzionato sito web, senza ovviamente trascurare le indicazioni della dottrina.

\section{Il sistema delle misurazioni}

\subsection{Il Bilancio preventivo e il Bilancio assestato}

Il Bilancio preventivo è un documento espresso in termini descrittivi e monetari che accoglie i costi e i ricavi di competenza dell'esercizio a cui si riferisce. Da qui, un punto di forza e uno di debolezza.

Più precisamente, il documento in questione, riferito al 2011, si apre con una relazione molto dettagliata del Presidente della Fondazione.

In essa, per quanto a noi interessa, si illustrano decisioni assunte nell'anno in corso che produrranno effetti su quelli successivi ${ }^{7}$, si esplicitano future iniziative e si riportano commenti e chiarimenti in merito a date voci di bilancio.

Il principio della trasparenza, che rimanda al concetto di accountability, trova ulteriore accoglimento quando, nella parte finale della relazione, si riportano alcune tabelle che evidenziano:

- i costi cosiddetti delegati dal Comune di Faenza alla Fondazione e i relativi contributi (presenti solo con riferimento al personale comunale “distaccato" presso il M.I.C) a carico dell'amministrazione comunale;

- la somma da essa stanziata a sostegno delle attività istituzionali del Museo;

- il riepilogo dei dati e il loro saldo finale, cioè i costi delegati che non risultano coperti da contributi a carico del Comune di Faenza e a cui,

\footnotetext{
${ }^{7}$ Valga, come esempio, la riduzione dei costi di energia elettrica, gas e sorveglianza dovuta, ed è solo una causa, al nuovo orario di apertura nel periodo invernale.
} 
pertanto, dovrà provvedere la Fondazione con risorse proprie onde assicurare il pareggio di bilancio.

Ancor più interessante è la precisazione dell'obiettivo, in precedenza segnalato, che ha ispirato la predisposizione del preventivo e che rimanda a un'oculata gestione dei costi. L'attenzione rivolta al contenimento dei costi trova una quantificazione quando si precisa che verrà presa in considerazione l'eventuale assunzione nell'organico della Fondazione di coloro che, alle dipendenze di terzi, svolgono il servizio di accoglienza e di biglietteria. Si stima, infatti, un risparmio che compenserà ampiamente i maggiori oneri fiscali. Trova così applicazione l'analisi costi/benefici che sempre deve guidare la decisione tra due o più alternative di scelta.

Chiara è, poi, l'applicazione del principio della prudenza nella quantificazione di taluni costi. Intendiamo riferirci all'incremento di valore della voce oneri e interessi passivi bancari, stante la previsione di possibili ritardi nell'incasso dei contributi delle amministrazioni pubbliche, e, per la sezione promozionale (di cui diremo tra poco), all'iscrizione in bilancio delle sole spese a fronte delle quali esistono entrate accertate e sufficienti alla loro copertura.

Tutto ciò detto, è evidente che la relazione in oggetto amplia la portata conoscitiva del preventivo in quanto, non solo fornisce informazioni su dati valori, ma ne esplicita le linee guida e le azioni (già in atto o programmate) che ne sono alla base. Ciò sarebbe ancor più vero se, come nel preventivo del 2009, fossero quantificati i costi riferiti ad attività promozionali che potrebbero essere realizzate in futuro nel caso in cui si trovasse la copertura finanziaria.

Il punto di debolezza sta nel fatto che, trattandosi di un preventivo economico, viene trascurata la dimensione finanziaria e quella patrimoniale. È quindi auspicabile che in futuro vengano considerate anche queste due dimensioni, in modo da poter disporre di uno strumento di programmazione riferito a tutti gli aspetti della gestione aziendale. Va, comunque, sottolineato che le dinamiche finanziarie e patrimoniali sono giornalmente monitorate, ma con lo scopo di prendere atto delle loro variazioni.

Il Bilancio di previsione è articolato, da un punto di vista spaziale, per macroattività e, talora, per attività.

Più precisamente, dei sette prospetti di cui si compone, due accolgono $\mathrm{i}$ costi e i ricavi della macroattività ordinaria e altri due i costi e i ricavi (contributi dei soci fondatori e dei sovventori) della macroattività promoziona$\mathrm{le}^{8}$ (i rimanenti prospetti riportano valori riepilogativi riferiti ad entrambe).

\footnotetext{
${ }^{8}$ Più precisamente, il verbale della seduta del Consiglio di Amministrazione del 5 luglio 2006 così si esprime: «[...] fanno parte della sezione ordinaria tutti quei ricavi e costi che,
} 
Se questa suddivisione, unitamente al grado di dettaglio nell'esposizione dei costi ordinari distinti per natura o per attività, è senz'altro da apprezzare sotto il profilo della capacità informativa del documento in esame, ancor più, a nostro avviso, lo è quella relativa ai costi della macroattività promozionale. Essi, infatti, sono classificati prevalentemente per destinazione quando vengono riferiti sia alle attività ricomprese sotto le voci: Eventi espositivi temporanei, Ricerche, studi e pubblicazioni, Attività di animazione sia alle voci Progetti europei "Interegg III", Spese promozionali e Costi gestione promozionale. Questi ultimi riguardano le spese postali e telefoniche iscritte per un importo pari, rispettivamente, all' $80 \%$ e al $70 \%$ del rispettivo totale.

Non esiste, invece, un'articolazione di tipo temporale, vale a dire per periodi infrannuali riferiti, ad esempio, alle quattro stagioni dell'anno, articolazione che, visti i tratti caratteristici dell'azienda museale, ben si addice ai valori di costo. A giustificazione di questa mancanza si adduce una certa rigidità che si verrebbe a creare nel caso, appunto, di una suddivisione in intervalli inferiori all'anno.

Il comportamento adottato può trovare una motivazione nel tipo di valori, di cui diremo tra breve, accolti nel preventivo, ma certamente non considera che talune attività (chiaro esempio è quella denominata Suggestioni Estive al M.I.C ) sono svolte solo in dati periodi dell'anno. Si rinuncia così $\mathrm{a}$ una guida più efficace, stante la variabilità della gestione nel tempo, e a interventi tempestivi allorquando sorgono date disfunzioni ${ }^{9}$.

La flessibilità al M.I.C è intesa quale assestamento (nel senso di "aggiornamento") in corso d'anno a cui, dal 2006, è sottoposto il Bilancio preventivo.

Nella riunione del Consiglio di Amministrazione del 5 luglio $2006 \mathrm{fu}$, infatti, approvata e resa operativa la proposta concernente l'assestamento del Bilancio preventivo. Nacque, così, il preventivo assestato, denominato Bilancio di assestamento.

consolidati nel tempo, sono necessari al regolare funzionamento del Museo ed i cui costi sono coperti da un contributo specifico da parte del Comune di Faenza o, in prospettiva, dal Governo centrale o locale, oltre che da ricavi di vendita (biglietti, ecc.)». Invece, «[...] fanno parte della sezione promozionale tutti quei ricavi e costi che afferiscono ad iniziative ed attività espositive, permanenti o temporanee, dotazioni strumentali, studi, ricerche, pubblicazioni, animazione e promozione, la cui attuazione derivi dalla programmazione, triennale o annuale ed i cui costi siano strettamente caratterizzati da un vincolo a copertura da parte di specifici e corrispondenti ricavi».

${ }^{9}$ È evidente che ciò è possibile solo se i costi consuntivi vengono rilevati con la stessa articolazione temporale scelta per quelli preventivi. 
Questo documento si apre con una parte descrittiva dove si precisa che le due sezioni di cui si compone saranno, rispettivamente, contraddistinte dagli aggettivi ordinaria e promozionale. Al fine di assegnare in modo appropriato $\mathrm{i}$ costi e i ricavi alle due sezioni, vengono formulate indicazioni di sintesi (si veda la nota 9) seguite dall'elenco di tutte le singole voci di costo e di ricavo.

Quanto ai valori del Bilancio assestato, riportati in prospetti sia analitici che sintetici, va in primo luogo puntualizzato che per ogni voce di costo e di ricavo viene indicato l'importo presente nel preventivo iniziale e quello assestato, riferendoli entrambi all'intero anno. Segue l'eventuale differenza tra i due valori con la relativa spiegazione.

In secondo luogo, va sottolineato che il documento in questione rappresenta una riformulazione, in corso d'anno, del Bilancio preventivo in quanto, sulla base degli accadimenti effettivi e della revisione dei dati previsionali, si sostituiscono valori consuntivi o accertati a valori preventivi e si correggono i valori futuri rendendoli più attendibili e, quindi, più utili ${ }^{10}$. È così che si può parlare di preconsuntivo e di due tipi di scostamenti, l'uno già verificatosi (e quindi in linea con il meccanismo di feed-back) e l'altro (fondato sul meccanismo di feed-forward) che presumibilmente si verificherà.

Interessante è il confronto tra il primo Bilancio di assestamento e quello del 2010.

Più in particolare, il secondo documento riporta una precisazione, assente nel primo, che, a nostro avviso, chiarisce la natura dei valori accolti nei due tipi di preventivo. Si afferma, infatti che l'assestamento è ritenuto «[...] utile al fine di evidenziare una tendenza sull'andamento della gestione del museo».

Inoltre, l'esposizione dei costi della gestione promozionale, strutturata come quella del preventivo del 2011, offre immediate e più chiare informazioni rispetto a quelle desumibili dal Bilancio assestato del 2006 dove, tra l'altro, non viene operata la ripartizione delle spese postali e telefoniche tra le due macroattività che connotano il Museo.

Nei prospetti numerici, invece, non vengono più riportate le differenze, con la relativa motivazione, tra i valori del Bilancio preventivo e quelli dell'assestato, informazioni queste che si sarebbero tradotte, perlomeno in taluni casi, in utili notizie per la gestione futura.

\footnotetext{
${ }^{10}$ Un chiaro esempio dell'indiscussa utilità di operare un'adeguata revisione del preventivo iniziale lo troviamo nel Bilancio assestato del 2010 dove figura un numero di mostre pari a circa il triplo di quelle indicate nel Bilancio preventivo dello stesso anno.
} 
Qualsiasi preventivo perderebbe parte della sua utilità se i suoi dati non venissero confrontati con quelli effettivi. È così che nel Bilancio consuntivo del $2010^{11}$ i valori del Preventivo assestato nello stesso anno vengono posti a raffronto con i rispettivi costi sostenuti e ricavi conseguiti avvalendosi, come per i bilanci descritti in precedenza, di quattro prospetti di cui due riferiti alla gestione ordinaria e due a quella promozionale ${ }^{12}$.

Completa questa informativa, oltre a precisazioni in merito ad alcune voci di bilancio, la spiegazione di taluni scostamenti registrati. Da non trascurare, poi, l'indicazione dei valori a consuntivo dei costi delegati dal Comune di Faenza, dei contributi da esso erogati e, per l'anno in oggetto, del totale degli oneri a carico della Fondazione non coperti da specifici contributi dell'Amministrazione comunale.

Infine, per quanto riguarda la funzione del preventivo quale strumento che guida e responsabilizza gli operatori aziendali verso obiettivi di breve periodo coerenti e strumentali a quelli definiti nell'ambito del piano strategico, occorre fare alcune osservazioni.

La prima è di ordine temporale. Più precisamente, il Bilancio preventivo deriva da una pianificazione triennale suddivisa, come sappiamo, in singoli anni.

La seconda riguarda la natura dei dati di costo che sono in prevalenza valori previsti e non importi programmati sulla base di prestabiliti livelli di efficienza, nell'acquisizione e nell'impiego delle risorse, ritenuti raggiungibili e, come tali, motivanti. Così operando, ed è quanto auspichiamo, verrebbero poste le premesse per il contenimento dei costi consuntivi. Inoltre, abbiamo già puntualizzato che certi costi vengono quantificati in maniera strettamente prudenziale; comportamento questo che può coniugarsi con quello, più generale, da noi suggerito. Infine, il ricorso a valori previsti e, talora, al principio della prudenza non toglie, tuttavia, che nel corso dell'anno gli operatori del Museo non cerchino di risparmiare su taluni oneri spuntando, ad esempio, prezzi minori, rispetto a quelli prospettati, nell'acquisto di materiali.

L'ultima considerazione intende precisare che i costi previsti sono concepiti come un vincolo da rispettare, stante l'imperativo di non sforare il bilancio $^{13}$, e come tali, e ancor più se fossero costi programmati, rappresentano

\footnotetext{
${ }^{11}$ Questo bilancio accompagna quello di esercizio redatto secondo la normativa vigente.

${ }^{12}$ A seguire, vi è un altro prospetto dove sono indicati gli investimenti lordi (immobilizzazioni materiali e immateriali) al 31 dicembre 2010.

${ }^{13}$ Emblematica in questo senso è l'affermazione del Presidente del M.I.C secondo cui la finalità alla base del sistema delle misurazioni è «[...] il raggiungimento degli obiettivi mantenendo il pareggio di bilancio». Ciò rimanda all'opinione della dottrina secondo cui il
} 
valori atti a guidare e responsabilizzare gli operatori del Museo. Relativamente alle attività che dipendono dalle scelte della dirigenza, il Bilancio preventivo ha come obiettivo anche quello di spingere gli alti livelli a conseguire, laddove è possibile, risparmi di costo. Qualora poi in corso d'anno si prefigurasse un onere più elevato, il surplus viene evidenziato per iscritto; dopodiché segue l'autorizzazione o il divieto a sostenere tale maggior onere.

Concludendo, il Bilancio preventivo e quello assestato del M.I.C, pur con i loro limiti, rappresentano apprezzabili strumenti di guida dei soggetti aziendali. Degni di particolare nota sono, poi, i confronti che tali documenti consentono di operare e, quindi, gli scostamenti di varia natura che mettono in evidenza, esaltando così il momento della verifica che tanta importanza ha nel controllo di gestione.

\subsection{I costi}

Per rispondere al secondo quesito di ricerca - che, lo ricordiamo, concerne anzitutto i problemi che si incontrano nell'impostare un sistema di rilevazione dei costi - ci baseremo, ma non in via esclusiva, su tre documenti: il consuntivo del 2010, il Bilancio assestato dello stesso anno e il preventivo del 2011. Inoltre, visto che le elaborazioni di costo riguardano la gestione sia promozionale che ordinaria, saranno proprio i prospetti degli oneri ad esse relativi quelli a cui faremo riferimento.

I costi della macroattività promozionale vengono attribuiti prevalentemente e secondo criteri di specialità (essendo costi diretti) sia ad ogni specifica attività che rientra nelle già ricordate categorie (Eventi espositivi temporanei, Ricerche, studi e pubblicazioni, Attività di animazione) sia alla voce Spese promozionali ${ }^{14}$.

Fanno eccezione:

- i costi associati ai Progetti europei "Interegg III" che configurano il

mantenimento dell'equilibrio economico-finanziario rappresenta, nelle aziende non profit, un vincolo da rispettare e non un obiettivo da conseguire (Matacena, 1999, p. 25; Santi, 2002, pp. 39-40; Marcon e Tieghi, 2000, p.79).

${ }_{14}^{14}$ I costi ricompresi nella voce Spese promozionali e sostenuti per la generalità degli eventi espositivi sono diretti anche se, ad esempio, sono relativi a un'inserzione pubblicitaria fatta per più mostre; nei confronti di ciascuna di esse sono invece indiretti. Gli altri costi promozionali - come, ad esempio, quelli sostenuti esclusivamente per una mostra (oneri per l'allestimento, per il catalogo, per i depliant e via dicendo) - sono diretti rispetto a detta mostra. 
costo complessivo il quale fa riferimento diretto al budget assegnato dal progetto europeo;

- gli oneri imputati globalmente, e quindi senza alcuna distinzione, alla voce relativa alle mostre minori, ai residui dell'anno 2009 o 2010 (a seconda del bilancio considerato) e a costi generali di tutti gli eventi espositivi temporanei;

- i costi della gestione promozionale ovvero le spese postali e telefoniche che, come già accennato, sono attribuite, essendo facilmente identificabili e suddivisibili, alla macroattività promozionale per un importo pari, rispettivamente, all' $80 \%$ e al $70 \%$ (a quella ordinaria, pertanto, vengono imputati valori pari al $20 \%$ e al $30 \%$ ). A questo riguardo, si nota un miglioramento visto che nel Bilancio di gestione del 2010 le spese in oggetto erano state attribuite per la metà a ciascuna delle due macroattività.

Quanto ai costi di ogni evento espositivo, che abbiamo appena visto essere tutti diretti, va puntualizzato che in un caso viene elaborata una configurazione più ampia. Questo accade quando si redige il rendiconto da presentare ai finanziatori; agli oneri diretti, infatti, viene aggiunta una quota della remunerazione del curatore delle mostre calcolata tenendo conto, in via approssimativa, delle giornate che questi ha dedicato a ciascuna di esse.

Nella relazione del Presidente del Consiglio di Amministrazione, inserita nel consuntivo del 2010, si precisa che «[...] tutte le altre spese ... (pulizie locali, alcune utenze come gas e luce, i servizi esterni di gestione della biglietteria, la sorveglianza e il costo del personale) sono state indicate nella gestione ordinaria anche se occorre evidenziare che molte di queste sono state sostenute in funzione e supporto alle manifestazioni svolte e andrebbero imputate pro quota» alla gestione promozionale, «[...] suddivisione questa che concretamente non si ritiene opportuno effettuare in quanto risulterebbe oltre che molto laboriosa soggetta a valutazioni alquanto discrezionali nella determinazione della percentuale di attribuzione» ${ }^{15}$.

La laboriosità e la soggettività nella ripartizione dei costi in oggetto alle due macroattività sono senz'altro condivisibili. D'altra parte, la discrezionalità nelle rilevazioni di costo, soprattutto se trattasi di costo complessivo, è ineliminabile e non può, se non in casi molto particolari, costituire motivo per sottrarsi a dette rilevazioni. Semmai occorre adoperarsi per trovare basi di imputazione degli oneri indiretti quanto più possibile aderenti al principio funzionale (Coda, 1968, p. 232 e ss.); in mancanza di queste, si dovrà

\footnotetext{
${ }^{15}$ Le voci di costo indicate tra parentesi vanno considerate come esempi.
} 
ripiegare su basi convenzionali. Inoltre, è necessario essere sempre consapevoli del grado di soggettività insito nelle elaborazioni di costo.

Non va, tuttavia, dimenticato che tutti o alcuni costi addossati per intero alla gestione ordinaria, ma di competenza anche di quella promozionale, verrebbero ugualmente e per lo stesso importo sostenuti anche in mancanza delle attività espositive temporanee; ne consegue che il loro costo si configura quale costo diretto suppletivo, valore, questo, utile per date decisioni e per finalità di controllo.

Un tentativo di addivenire a una configurazione più ampia del costo diretto delle singole mostre fu fatto nel 2008 e descritto nel Bilancio consuntivo dello stesso anno.

Più precisamente, al costo diretto di ogni manifestazione fu sommata una quota degli oneri promozionali sostenuti per tutte le mostre, quota ottenuta utilizzando come base di riparto il costo diretto di ciascuna di esse. Tali oneri indiretti vennero così ripartiti «[...] con criteri soggettivi e sulla base dell'effettiva onerosità sostenuta di ogni manifestazione».

Detta procedura fu abbandonata in quanto si ritenne che avrebbe fatto perdere di vista l’intera attività del Museo.

Per quanto riguarda le attività della gestione ordinaria - come il restauro, il laboratorio Giocare con l'Arte e via dicendo - vengono elaborati costi parziali quale, ad esempio, l'onere sopportato per i materiali impiegati nel laboratorio di restauro.

In conclusione, le scelte relative agli oggetti di costo e ai corrispondenti oneri imputati possono così sintetizzarsi:

- macroattività ordinaria: costi diretti + quota parte di taluni oneri indiretti (spese postali e telefoniche) + totale dei rimanenti costi indiretti;

- macroattività promozionale: costi diretti + quota parte di taluni oneri indiretti (spese postali e telefoniche);

- singole attività della gestione ordinaria: costi parziali;

- singole attività della gestione promozionale: costi diretti $(+$, per certi fini, quota parte di un onere indiretto) e costo complessivo per il citato progetto europeo.

La metodologia adottata per la rilevazione dei costi è molto semplice in quanto per l'imputazione degli oneri indiretti non si ricorre, quale destinazione intermedia nell'allocazione dei costi, né ai centri di costo né alle attività (Agliati, 1995; Bartolini e Visani, 2004; Brusa, 1995; Bubbio, 2001; Cinquini, 2008; Cinquini, 2009; De Rosa, 2000; Gosselin, 2007; Zanigni, 
2004), ma ci si basa sulla ripartizione tra le due macroattività delle spese postali e telefoniche secondo percentuali forfettariamente definite.

Sotto il profilo temporale, la rilevazione dei costi è, tranne alcune eccezioni, su base annuale (si tratta, infatti, degli stessi oneri presenti nei preventivi e nel consuntivo) e, considerato il momento in cui si effettua il calcolo, trattasi di valori preventivi, consuntivi e preconsuntivi.

Infine, il sistema di rilevazione non fa uso della partita doppia e, pertanto, è di tipo extracontabile; come tale, presenta una flessibilità che consente di addivenire a ogni tipo di costo.

Da quanto fin qui esposto, ivi comprese le considerazioni fatte in merito ai bilanci, emerge evidente che le elaborazioni di costo rispondono a finalità decisionali e di controllo. In questo secondo caso, i costi rappresentano una guida per gli operatori museali e consentono di operare adeguate verifiche in corso d'anno e alla fine dello stesso.

Per quanto concerne, in particolare le singole attività promozionali, ricomprese nelle tre categorie più volte richiamate, conoscerne il costo è molto importante in quanto la sua entità non deve superare l'ammontare prestabilito dei contributi dei privati.

Infine, ricordiamo il costo che viene calcolato quando si redige il rendiconto da presentare ai finanziatori delle mostre.

\subsection{Gli indicatori quantitativo-non monetari e qualitativi}

Gli indicatori fisico-tecnici e qualitativi ${ }^{16}$, e affrontiamo così la prima parte dell'ultimo quesito di ricerca, trovano ampio spazio in un documento, con un'accattivante veste grafica e ricchissimo di notizie, che è denominato Resoconto Attività. Anche quello che riguarda il 2010 contiene, tra le tante informazioni, una serie di indicatori che gettano luce sulla vasta e poliedrica attività del M.I.C.

Più in particolare, le misurazioni riguardano:

1. i visitatori. Avvalendosi di varie forme espositive (ad esempio, grafici e istogrammi) vengono evidenziati, anno per anno e a partire dal 2004 o da anni successivi e sempre fino al 2010, numerosi dati, talvolta rapportati tra di loro, relativi agli ingressi (suddivisi anche per mese, giorno e ora), ai visitatori in gruppo, alle tipologie, con il relativo numero, di biglietti venduti;

\footnotetext{
${ }^{16}$ Gli indicatori in oggetto trovano ampio accoglimento nella Balanced Scorecard (Marcon, 2004, p. 30 e ss.).
} 
2. il restauro. Dalle pagine del Resoconto, che riportano una o più fotografie degli oggetti restaurati, si evince il numero degli interventi effettuati nel 2010 ad opere appartenenti alle collezioni del M.I.C. Viene, poi, indicato il numero dei restauri eseguiti per committenti esterni;

3. il sito web. Espressi da grafici, tabelle e via dicendo, i dati relativi alla consultazione, sia del vecchio che del nuovo sito, mettono in evidenza, in valore assoluto e/o in percentuale, le "sorgenti" del traffico, le visualizzazioni di pagina e per alcune di esse i principali contenuti, le pagine per visita (valore medio), il tempo medio di permanenza sul sito, le nuove visite e la frequenza di rimbalzo. La maggior parte di questi dati sono, poi, disaggregati per nazione (10 su un totale di 87);

4. le attività didattiche. Delle molte misurazioni basti ricordare il numero di: interventi realizzati, utenti, gruppi e/o classi (a cui si aggiunge la media dei componenti). Talora il totale degli utenti è suddiviso in base alla loro provenienza territoriale e ai vari ordini di scuole;

5. la biblioteca. Per ogni anno dal 2000 al 2010, si trova l'indicazione delle presenze in biblioteca, divise tra persone fisiche che hanno fatto le loro ricerche all'interno della biblioteca stessa e utenza esterna (prestiti interbibliotecari e servizi di document delivery), e del numero dei volumi consultati. A ciò si aggiungono altri dati come il numero delle nuove accessioni inventariate e catalogate dal 2001 al 2010;

6. la fototeca relativamente alla quale si riportano alcuni dati tra cui il numero delle unità (fotografie, diapositive e via dicendo) ancora da archiviare;

7. altre attività; ad esempio, esposizioni temporanee, pubblicazioni curate dal Museo, concerti aperitivo, il cui numero si deduce dalle illustrazioni presenti nel Resoconto.

Pur essendo succinta e talora parziale, la trattazione degli indicatori sopra riportata denuncia chiaramente quanto essi siano numerosi ed eterogenei. Apprezzabile è poi la forma espositiva, variegata e capace di fornire un'immediata percezione dei dati, oltre all'elevato grado di analisi, che connota alcune misurazioni, e all'indicazione di valori riferiti a più anni in grado, come tali, di evidenziare il trend delle dinamiche poste sotto osservazione.

Per completare il quesito di ricerca, che prevede anche indicatori qualitativi, va osservato che essi sono rappresentati dai giudizi e dai suggerimenti riportati dai visitatori nel registro e in appositi foglietti di carta messi a loro disposizione presso il bookshop. 
Tutti gli indicatori evidenziati sono ritenuti interessanti per rilevare il gradimento, da parte degli utenti del prodotto M.I.C e dei servizi che il Museo offre. Tra queste misurazioni ve ne sono alcune impiegate per formulare decisioni future o per esprimere un parere sugli investimenti effettuati con particolare riguardo ai relativi costi.

Inoltre, le informazioni sul sito web sono apprezzate anche quando occorre esprimere un giudizio sulla convenienza o meno a investire ulteriormente in questo strumento di comunicazione.

Infine, i dati sulle attività didattiche sono considerati importanti perché completano la generale finalità conoscitiva sopra accennata quando vengono utilizzati per capire quali sono le attività preferite dagli utenti.

\section{Verso un miglioramento}

Gli strumenti conoscitivi presi in esame sono suscettibili di essere affinati in modo tale da aumentarne la portata informativa e di renderli più idonei al perseguimento delle finalità a cui sono diretti. In precedenza, ci siamo già soffermati su alcuni interventi in questo senso. Resta, pertanto, da completare il quadro con nuovi suggerimenti.

Il Bilancio preventivo vedrebbe aumentata la sua capacità informativa se, come nel 2009, accogliesse anche i costi riferiti ad attività promozionali realizzabili in futuro nel caso in cui si trovasse la copertura finanziaria. Similmente dicasi in merito al ripristino nel Bilancio assestato delle due colonne dove vengono indicate le differenze, con la relativa motivazione, tra $\mathrm{i}$ valori in esso inseriti e quelli del preventivo.

Come per i bilanci, anche per i costi - che sono poi quelli ricompresi in detti documenti - è opportuna una elaborazione infrannuale.

Un considerevole miglioramento si avrebbe dall'adozione dell'Activity Costing e, ancor più, dell' Activity Based Costing che richiede però dei cambiamenti rispetto alla metodologia più conosciuta e concepita per le aziende di produzione diretta di beni. Un'altra soluzione potrebbe essere quella della contabilità per centri di costo (Mazzotta, 2006, p. 279 e ss.; Puntillo, 2006, p. 338 e ss.).

Il primo metodo prevede l'attribuzione dei costi a ogni attività del $\mathrm{Mu}$ seo, dopo averle adeguatamente individuate. Alcune sono rintracciabili nei bilanci esaminati; ci riferiamo alle attività promozionali e a quelle esternalizzate. Delle restanti, che richiedono la conoscenza dell'intera attività del 
Museo, ricordiamo: l'esposizione permanente, il restauro, l'attività didattica e formativa, le conferenze e i convegni, l'approvvigionamento dei materiali e l'attività di segreteria.

Alcune di dette attività sono scomponibili in attività più elementari. Ad esempio, nella Carta dei servizi del M.I.C la formazione e la didattica viene suddivisa in: attività didattica, formazione permanente agli adulti, tirocini e stage, assistenza tesi di laurea, formazione per insegnanti. Riteniamo tuttavia che, quanto meno a un primo approccio, sia bene non spingersi fino a un grado d'indagine così profondo e che l'analisi costi/benefici debba sempre guidare la scelta del livello di approfondimento da riservare alle rilevazioni di costo.

Individuate le attività, occorre imputare a ciascuna di esse i costi di tutti i fattori produttivi impiegati, ad eccezione, per motivi diversi, di quelle esternalizzate, di cui già si conosce in gran parte o l'intero onere sopportato, e del personale "distaccato" dal Comune di Faenza e dalla Regione Emilia Romagna la cui remunerazione, come sappiamo, è a carico del rispettivo ente locale.

Se per i costi diretti non esistono particolari problemi di allocazione (si pensi alla remunerazione dei restauratori e all'onere sopportato per i materiali utilizzati dalle varie attività), per quelli indiretti occorre trovare adeguati resource driver. Ad esempio, il costo dell'energia elettrica per illuminazione potrebbe essere ripartito in base ai metri quadri o ai metri cubi dei locali dove si svolgono le attività.

Un passo in avanti nella rilevazione dei costi consiste nell'imputare gli oneri delle attività di supporto a quelle che costituiscono l'output globale del Museo. Sempre esemplificando, il costo dell'approvvigionamento dei materiali potrebbe essere suddiviso tra le attività che ne hanno beneficiato tramite l'activity driver rappresentato dal numero degli ordini di acquisto.

Per finire, anche l'insieme degli indicatori fisico-tecnici e qualitativi nonché il loro impiego si prestano a miglioramenti (Ames, 1994; Catalfo et al., 2007, p. 205 e ss.; Dainelli, 2007, p.146 e ss; Weil, 1994).

Oltre a comparazioni nel tempo, già accolte dal M.I.C, si potrebbero effettuare confronti sia tra valori obiettivo e valori consuntivi, per verificare il grado di aderenza ai programmi, sia con alcuni musei dello stesso tipo, al fine di trarre utili indicazioni per il miglioramento della performance aziendale (Solima, 2009, pp. 505-506). In questo secondo caso, se sono le best practices ad essere scelte, si prefigura una vera e propria attività di benchmarking.

La compilazione, da parte dei visitatori, di un questionario ben strutturato potrebbe consentire la costruzione di nuovi indicatori e completare quan- 
to emerge dagli strumenti attualmente utilizzati per raccogliere i giudizi e i suggerimenti dei visitatori; sarebbe poi opportuno sostituire detti strumenti con apposite domande nel questionario e con una parte dello stesso, lasciata in bianco, intitolata "Giudizi e suggerimenti".

Esistono, infatti, altri indicatori riguardanti i visitatori che non vanno sottovalutati soprattutto quando sono capaci di delinearne il profilo e il grado di soddisfazione. Ad esempio, tali sono: il sesso, l'età, l'attività professionale, il titolo di studio, le visite ad altri musei negli ultimi dodici mesi, le sensazioni provate durante la visita (accrescimento culturale, piacere estetico, curiosità e via dicendo), i giudizi su più aspetti come l'orario di apertura e l'accoglienza alla biglietteria, l'illuminazione nelle sale.

Naturalmente, il questionario deve essere adeguatamente dimensionato e articolato ${ }^{17}$; e fu proprio per la sua lunga compilazione che il questionario a cui fece ricorso il M.I.C non venne favorevolmente accolto dai visitatori e per questo abbandonato sebbene ritenuto utile a fini statistici.

Merita, comunque, segnalare che per la valutazione della crescita culturale non è sufficiente l'intervista, ma occorrono altre tecniche (Dean, 1996; Kotler e Kotler, 1998), come quelle di matrice psico-sociologica, che richiedono tempo e risorse adeguate che il M.I.C, data l'attuale crisi che presumibilmente investe anche i suoi finanziatori, forse non è in grado di procurarsi.

Per quanto concerne le finalità dei singoli indicatori, non ravvisiamo un'organicità di utilizzo; pertanto, è auspicabile un completo e più articolato impiego delle informazioni che essi sono in grado di trasmettere.

Per concludere, sarebbe certamente di grande aiuto per il M.I.C fare riferimento alla Scheda n. 31 delle "Linee guida per la redazione del bilancio sociale delle organizzazioni non profit”. In essa, infatti, si riporta un'ampia e ben articolata gamma di indicatori (quantitativo-monetari, come i costi degli eventi speciali e il costo medio per singolo visitatore, fisico-tecnici e qualitativi) raggruppati a seconda dell'obiettivo conoscitivo e riferiti a: collezioni e opere, attività, utenti, risorse per l'attività museale, indici economici specifici dell'attività, soddisfazione e qualità.

Nell'ottica sistemica a cui abbiamo fatto riferimento a proposito delle domande di ricerca, va evidenziato che:

\footnotetext{
${ }^{17}$ Un suggerimento potrebbe essere quello di inserire un primo insieme di domande destinate a tutti i visitatori seguite da altre che si differenziano a seconda che siano rivolte, ad esempio, a bambini, ad adulti, a studiosi.
} 
- considerando le macroattività (ordinaria e promozionale) e le singole attività della gestione promozionale, le "figure" di costo si ritrovano, oltre che nel Bilancio preventivo e in quello assestato, anche nel Bilancio consuntivo;

- ai prospetti che accolgono i costi e i ricavi effettivi nonché i corrispondenti valori desunti dal preventivo assestato segue la spiegazione di taluni scostamenti registrati;

- dalla lettura del Resoconto Attività emergono, talora, le cause dell'ammontare di dati costi e ricavi. Quanto ai primi, ne sono esempi il numero delle opere esposte in alcune mostre temporanee e il numero delle attività di intrattenimento musicale distinte in Concerti Aperitivo e Suggestioni Estive al MIC. Riguardo ai secondi, e sempre esemplificando, ricordiamo l'orario di apertura e chiusura del Museo e le tipologie, con il relativo numero, di biglietti venduti; queste notizie spiegano, seppur in parte, l’importo derivante dagli Ingressi al Museo.

In conclusione, i miglioramenti che abbiamo proposto non sono pochi e, talora, richiedono molto tempo. Siamo, tuttavia, dell'avviso che nel M.I.C vi siano le competenze adatte per affinare e ampliare, in modo graduale, il sistema delle misurazioni e renderlo così sempre più in grado di supportare gli operatori museali nelle continue sfide che provengono dall'ambiente esterno.

\section{Bilbiografia}

AA.VV., (2008), Siti della ceramica dell'euro regione adriatica: catalogazione informatizzata, conservazione, gestione e valorizzazione, Oria (Br), Italgrafica Edizioni.

AGLIATI M., (1995), Costi di prodotto e controllo dei costi: dai sistemi tradizionali al metodo basato sulle attività, in F. Amigoni (a cura di), "Misurazioni d'azienda. Programmazione e controllo", Milano, Giuffrè.

AGLIATI M., (2003), Condizioni di efficacia nelle misure non finanziarie nella valutazione delle prestazioni aziendali, in F. Amigoni, P. Miolo Vitali (a cura di), "Misure multiple di performance”, Milano, Egea.

AMES P. J., (1994), Measuring museums 'merits', in Moore K. "Museum management”, Londra, Routledge.

BARTOLINI M., VISANI F., (2004), L'Activity-Based Costing: dalla teoria alla pratica, in "Budget", n. 40.

BERNARDI B., (2004), Realizzare la mission delle istituzioni culturali attraverso il sistema di programmazione e controllo, in B. Sibilio Parri (a cura di), "Misurare e comunicare i risultati. L'accountability del museo", Milano, F. Angeli.

BRUNETTI G., (1993), Il sistema di reporting e le quantità non monetarie, in "Studi in onore di Carlo Masini”, vol. III, Milano, Egea. 
BRUSA L., (1995), Contabilità dei costi. Contabilità per centri di costo e Activity Based Costing, Milano, Giuffrè.

BRUSA L., (2000), Sistemi manageriali di programmazione e controllo, Milano, Giuffrè.

BUBBIO A., (2001), Calcolo dei costi per attività, Milano, Guerini e Associati.

CARNEGIE G.D., WOLNIZER P.W., (1996), Enabling accountability in museums, in “ Accounting, Auditing \& Accountability Journal”, vol. 9, n. 5.

CATALFO P., CIURCINA C., CARDILLO E., DI DIO G., MOLINA S., (2007), Gestione e controllo del patrimonio immateriale negli enti culturali: alcune evidenze empiriche nei processi di misurazione del valore nelle aziende museali, in B. Sibilio Parri (a cura di), "Responsabilità e performance nei musei”, Milano, F. Angeli.

CATALFO P., MOLINA S., (2004), La cultura dell'accountability nei musei: misurare le performances per comunicare il valore, in B. Sibilio Parri (a cura di), "Misurare e comunicare i risultati. L'accountability del museo”, Milano, F. Angeli.

CINQUINI L., (2008), Strumenti per l'analisi dei costi. Fondamenti di cost accounting, vol. I, Torino, Giappichelli.

CINQUINI L., (2009), Aspetti critici dell'activity-based costing come sistema progredito di calcolo dei costi, in P. Miolo Vitali (a cura di), "Strumenti per l'analisi dei costi”, vol. II, “Approfondimenti di cost accounting”, Torino, Giappichelli.

CODA V., (1968), I costi di produzione, Milano, Giuffrè.

DAINELLI F., (2007), Il sistema di programmazione e controllo del museo, Milano, F. Angeli.

DEAN D., (1996), Museum Exhibition, London, Routledge.

DE ROSA B., (2000), Attività aziendali e processi di attribuzione dei costi. Un'analisi critica dell'ABC, Trieste, Edizioni Goliardiche.

DEL SORDO C., LEVY ORELLI R., PAZZI S., (2010), Dal "pubblico"al non profit. L' esperienza del Museo Internazionale delle Ceramiche di Faenza, in "Non profit”, n. 2.

FERRETTI M., (2007), Finanziamento e performance nei musei: casi di studio, in B. Sibilio Parri (a cura di), "Responsabilità e performance nei musei”, Milano, F. Angeli.

GARRISON R.H., NOREEN E.W., (2004), Programmazione e controllo. Managerial accounting per le decisioni aziendali, Milano, McGraw-Hill.

GIACINTI R., (2010), Un sistema di indicatori per valutare le performance degli enti non profit, in "Il Sole 24 Ore. Terzo Settore".

GOSSELIN M., (2007), A review of activity-based costing: technique, implementation, and consequences, in C.S. Chapman, A.G. Hopwood, M.D. Shields (eds), "Handbook of Management Accounting Research”, vol. II, Elsevier.

HORNGREN C.T., BHIMANI A., DATAR S.M., FOSTER G., (2002), Management and cost accounting, London, Prentice Hall.

ITTNER C.D., LARKER D.F., (2003), Coming up short on nonfinancial performance measurement, in "Harvard Business Review", November.

KOTLER N., KOTLER P., (1998), Museum strategy and marketing, San Francisco, Jossey-Bass.

MARCON G., (2004), La gestione del museo in un'ottica strategica: l'approccio della balanced scorecard, in B. Sibilio Parri (a cura di), "Misurare e comunicare i risultati. L'accountability del museo", Milano, F. Angeli.

MARCON G., TIEGHI M., (2000), Sistema informativo e misurazioni economiche nelle aziende non profit, in A. Zangrandi (a cura di), "Aziende non profit. Le condizioni di sviluppo”, Milano, Egea.

MATACENA A., (1999), Introduzione: la rete concettuale, in A. Matacena (a cura di), “Aziende non profit. Scenari e strumenti per il terzo settore”, Milano, Egea. 
MATACENA A., (2007), Mission, accountability e accreditamento nei musei: un percorso interpretativo, in B. Sibilio Parri (a cura di), "Responsabilità e performance nei musei", Milano, F. Angeli.

MAZZOTTA R, (2006), Il sistema di contabilità analitica nei musei, in B. Sibilio (a cura di), "Un modello di misurazione delle performance dei musei con particolare riferimento alla realtà calabrese”, Roma, Aracne.

McLANEY E., ATRILL T., (2002), Accounting. An introduction, London, Prentice Hall.

MOTEDURO F., (2004), L'accountability delle aziende museali: l'esperienza di Italia $e$ Regno Unito, in B. Sibilio Parri (a cura di), "Misurare e comunicare i risultati. L'accountability del museo”, Milano, F. Angeli.

PUNTILLO P., (2006), L'analisi e la rilevazione dei costi nei musei: una proposta metodologica per la Galleria Nazionale di Palazzo Arnone, in B. Sibilio (a cura di), "Un modello di misurazione delle performance dei musei con particolare riferimento alla realtà calabrese”, Roma, Aracne.

RENTSCHLER R., POTTER B., (1996), Accountability versus artistic development. The case for non-profit museums and performing arts organizations, in " Accounting, Auditing \& Accountability Journal”, vol. 9, n. 5.

ROCCHI F., (2004), Missione e scelte strategiche, in B. Sibilio (a cura di), "Definire la missione e le strategie del museo", Milano, F. Angeli.

ROCCHI F., (2007), Vision e mission nella strategia del museo, in B. Sibilio Parri (a cura di), "Responsabilità e performance nei musei”, Milano, F. Angeli.

RUGGIERI M., (2004), I costi aziendali: strumenti di calcolo e logiche di gestione tra tradizione e innovazione, Milano, Giuffrè.

SANTI M., (2002), Controllo di gestione per le aziende non profit, sanitarie e pubbliche. Verso la comunicazione multidimensionale, Milano, Egea.

SIBILIO PARRI B., (2004), Quale bilancio per il museo?, in B. Sibilio Parri (a cura di), "Misurare e comunicare i risultati. L'accountability del museo", Milano, F. Angeli.

SIBILIO PARRI B., (2007), La rendicontazione e la comunicazione dell'attività museale, in B. Sibilio Parri (a cura di), "Responsabilità e performance nei musei”, Milano, F. Angeli.

SIBILIO PARRI B., (2011), Strumenti di accountability dei musei: esperienze e proposte, in M. Montella, M. Cerquetti (a cura di), "Economia, cultura e territorio", Atti della giornata di studio, Fermo, Facoltà di Beni Culturali, 7 dicembre 2009, in "Quaderni del Dipartimento di Beni Culturali”, n. 1, Macerata , EUM.

SOLIMA L., (2009), Nuove metriche per comunicare il museo, in "Economia della Cultura”, n. 4.

WEIL S., (1994), Performance indicators for museums: progress report from wintergreen, in " Journal of Arts Management, Law and Society”, Winter.

ZANGRANDI A., (2007), Il finanziamento dei musei: condizioni per l'economicità e lo sviluppo, in B. Sibilio Parri (a cura di), "Responsabilità e performance nei musei”, Milano, F. Angeli.

ZANIGNI M., (2004), L'Activity-Based Costing in atto. Metodo, progettazione, implementazione, Padova, Cedam.

ZAPPA G., (1957), Le produzioni nell'economia delle imprese, vol. II, Milano, Giuffrè.

\section{Sitografia}

www.micfaenza.org 